FACTA UNIVERSITATIS (NIŠ)

Ser. Math. Inform. Vol. 34, No 4 (2019), 651-658

https://doi.org/10.22190/FUMI1904651G

\title{
ON THE PARTIAL DIFFERENCE SETS IN CAYLEY DERANGEMENT GRAPHS
}

\author{
Modjtaba Ghorbani* and Mina Rajabi-Parsa
}

(C) 2019 by University of Niš, Serbia | Creative Commons Licence: CC BY-NC-ND

\begin{abstract}
Let $G$ be a finite group. The set $D \subseteq G$ with $|D|=k$ is called a $(n, k, \lambda, \mu)$ partial difference set (PDS) in $G$ if the differences $d_{1} d_{2}^{-1}, d_{2}, d_{2} \in D, d_{1} \neq d_{2}$, represent each non-identity element in $D$ exactly $\lambda$ times and each non-identity element in $G-\{D\}$ exactly $\mu$ times. In the present paper, we determine for which group $G \in\left\{D_{2 n}, T_{4 n}, U_{6 n}, V_{8 n}\right\}$ the derangement set is a PDS. We also prove that the derangement set of a Frobenius group is a PDS.
\end{abstract}

Keywords. Finite group; Frobenius group; derangement set.

\section{Introduction}

Let $G$ be a finite group. A symmetric subset of group $G$ is a subset $S \subseteq G$, where $1 \notin S$ and $S=S^{-1}$. The Cayley graph $\Gamma=\operatorname{Cay}(G, S)$ with respect to $S$ is a graph whose vertex set is $V(\Gamma)=G$ and two vertices $x, y \in V(\Gamma)$ are adjacent if and only if $y x^{-1} \in S$. It is a well-known fact that a Cayley graph is connected if and only if $G=\langle S\rangle$. Also a Cayley graph is a regular graph (every vertex has the same degree).

A derangement is a permutation with no fixed points. The set $\mathcal{D}$ of permutation group is derangement if all elements of $\mathcal{D}$ are derangements. Suppose $G$ is a permutation group and $\mathcal{D} \subseteq G$ is a derangement set. The derangement graph $\Gamma_{G}=\operatorname{Cay}(G, \mathcal{D})$ has the elements of $G$ as its vertices and two vertices are adjacent if and only if they do not intersect.

Suppose $G$ is a permutation group of degree $n$. A subset $S$ of $G$ is said to be intersecting if for any pair of permutations $\sigma, \tau \in S$ there exists $i \in\{1,2, \ldots, n\}$ such that $\sigma \tau^{-1}(i)=i$. A group $G$ has the Erdös-Ko-Rado $(\boldsymbol{e k r})$ property, if for any intersecting subset $S \subseteq G,|S|$ is bounded above by the size of the largest point stabilizer in $G$. The maximal intersecting set is one with maximum size. A group can have the property under one action while it fails to have this property under

Received January 24, 2019; accepted July 05, 2019

2010 Mathematics Subject Classification. Primary 05C35; Secondary 05E30, 05B10 
another action. We refer to $[1,2,8,9,13,17]$ for background information about the history of this intresting problem.

Section 2 includes the $\boldsymbol{e} \boldsymbol{k r}$ properties of well-known groups. In section 3, the derangement set of well-known groups are studied.

\section{Erdös-Ko-Rado property}

For the subgroup $H$ of group $G$ and the element $g \in G$, the conjugate of subgroup $H$ in $G$ is denoted by $H^{g}=g^{-1} H g$. Suppose $G \leq \operatorname{Sym}(n)$ is a transitive permutation group, then $G$ is called a Frobenius group if it has a non-trivial subgroup $H$, where $H \cap H^{g}=\{1\}$, for all $g \in G \backslash H$. The kernel of Frobenius group $G$ is defined as

$$
K=\left(G \backslash \cup_{g \in G} H^{g}\right) \cup\{1\} .
$$

It is not difficult to see that all non-identity elements of $K$ are all derangement elements of $G$. In other words, let $G$ be a non-trivial permutation group and $G^{*}=G-\{1\}$. If $G$ is a Frobenius group then for all $g \in G^{*},|f i x(g)| \leq 1$ and at least there exist an element $g_{0} \in G^{*}$ such that $\mid$ fix $\left(g_{0}\right) \mid=1$.

Theorem 2.1. [16] (Frobenius Theorem) Suppose $H$ is a proper non-identity subgroup of $G$ such that for all $g \in G \backslash H$, we have $H \cap g^{-1} H g=\{1\}$. Let $K=G \backslash \cup_{g \in G} g^{-1}(H \backslash\{1\}) g$, then $K \triangleleft G, G=K H$ and $H \cap K=\{1\}$.

Proposition 2.1. [2] Every Frobenius group has the ekr property.

Theorem 2.2. Let $G \leq \operatorname{Sym}(n)$ and the derangement graph $C a y(G, \mathcal{D})$ be the disjoint union of $n$-cliques. Then $G$ has the ekr property.

Proof. Let $\left\{k_{1}, k_{2}, \ldots, k_{n-1}\right\}$ be the set of derangements of $G$ and $\left\{g_{i}, g_{i} k_{1}, \ldots, g_{i}\right.$ $\left.k_{n-1}\right\}$ be the vertices of the $i$-th clique in derangemen graph $\operatorname{Cay}(G, \mathcal{D})$, where $g_{i} \in G$. Since each clique has size $n$ and $G$ acts on $n$ elements, every elemen of each clique has exactly one fixed point and every pair of elements in a clique has no same fixed point. Let $H$ be the set of all vertices in $\operatorname{Cay}(G, \mathcal{D})$ that fixes point $x$. Suppose $1 \neq g_{r} k_{t} \in H$ and $\left(g_{r} k_{t}\right)^{g} \in H$, where $g \in G-H$. So $g^{-1} g_{r} k_{t} g(x)=x$ and thus $g_{r} k_{t} g(x)=g(x)$. This means that $g_{r} k_{t}$ fixes $g(x)$ while $g(x) \neq x$, a contradiction. The proof is completed.

A group $G$ acting on a set $X$ is transitive if for every pair of points $(a, b) \in X$ there exist $x \in G$ such that $x . a=b$. The permutation group $G$ is regular if $G$ acts transitively on $X$ and for all $x \in X, G_{x}=1$. A group $G$ is 2-transitive if for any two ordered pairs $(a, r),(b, s) \in X$, with $a \neq r$ and $b \neq s$ there exists $x \in G$ such that $x . a=b$ and $x . r=s$. We say that $G$ is sharply 2-transitive if $G$ is 2-transitive and for any two points $x, y \in X, G_{x, y}=1$. In this paper by, $(G \mid X)$ we mean that the group $G$ acts on the set $X$. 
Theorem 2.3. $\quad[5]$ Let $(G \mid X)$ be transitive and $x \in X$. Then $(G \mid X)$ is 2-transitive if and only if $G_{x}$ acts transitvely on the set $X-\{x\}$.

Theorem 2.4. [5] (The orbit-stabilizer property) Let $(G \mid X)$ and $x \in X$. If $G$ is finite, then $\left|x^{G}\right|\left|G_{x}\right|=|G|$.

Theorem 2.5. [5] (Galois Theorem). Let $(G \mid X)$ be a transitive permutation group of degree a prime number. Then the group $G$ is solvable if and only if for all $x, y \in X, x \neq y$, we have $G_{x, y}=1$.

Theorem 2.6. Let $(G \mid X)$ be a 2-transitive permutation group of degree $n$ and $\left(x_{1}, x_{2}\right) \in X^{2}$. Then $|G|=n(n-1)\left|G_{x_{1}, x_{2}}\right|$.

Proof. Suppose the group $G$ acts on $X$, transitively. So the action of $G$ on $X$ has one orbit. Then by Theorem 2.4, $|G|=n\left|G_{x_{1}}\right|$. On the other hand, by Theorem 2.3 group $G_{x_{1}}$ acts transitively on the set $X-\left\{x_{1}\right\}$, and by the orbit-stabilizer property $\left|G_{x_{1}}\right|=(n-1)\left|G_{x_{1}, x_{2}}\right|$. This completes the proof.

Theorem 2.7. Let $(G \mid X)$ be a transitive non-regular group of degree a prime number. If $G$ is solvable then $G$ has the ekr property.

Proof. Since $G$ is non-regular, there exist $x \in X$ such that $G_{x} \neq 1$. By Theorem 2.5 , for $x, y \in X$ we have $G_{x, y}=1$ and this means that every non-identity element of $G$ fixes at most one element. If every non-identity element of $G$ fixes no element of $X$, then $|G|=|X|$ and it is contradict with the non-regularity of $G$. So there exist at least one $1 \neq x \in X$ such that $\left|G_{x}\right|=1$. Hence, $G$ is Frobenius group and by Proposition 2.1, it has the $\boldsymbol{e} \boldsymbol{k r}$ property.

Theorem 2.8. Let $(G \mid X)$ be a transitive permutation group such that the action $G$ is non-regular and for all $x, y \in X, x \neq y$, we have $G_{x, y}=1$. Then $G$ has the ekr property.

Proof. Similar to the proof of theorm 2.7, we can conclude that $G$ is Frobenius group and the result follows.

Theorem 2.9. [5] Let $(G \mid X)$ and the act of $G$ be 2-transitive. Then the action of $G$ on $X$ is sharply 2-transitive if and only if $|G|=n(n-1)$.

Theorem 2.10. Let $(G \mid X)$ be 2-transitive non-regular permutation group of degree $n$ such that $|G|=n(n-1)$. Then $G$ has the ekr property.

Proof. By Theorem 2.9, $G$ is a sharply 2-transitive group and so for $x, y \in X(x \neq y)$, we have $G_{x, y}=1$. Now, similar to the proof of Theorem 2.7, $G$ is a Frobenius group and thus it has the $\boldsymbol{e} \boldsymbol{k} \boldsymbol{r}$ property. 
Let $\rho: G \rightarrow G L(n, \mathbb{F})$ be a representation with $\rho(g)=[g]_{\beta}$. The character $\chi_{\rho}: G \rightarrow \mathbb{C}$ of $\rho$ is defined as $\chi_{\rho}(g)=\operatorname{tr}\left([g]_{\beta}\right)$ for some basis $\beta$. The character $\chi$ of an irreducible representation is called the irreducible character and $\chi$ is linear, if $\chi(1)=1$. The set of all irreducible characters of group $G$ is denoted by $\operatorname{Irr}(G)$.

Let $(G \mid X)$ and $f i x(g)=\{x \in X \mid g(x)=x\}$. The character $\pi$ such that $\pi(g)=$ $|f i x(g)|$ is called permutation character and the character $\chi=\mid$ fix $(g) \mid-1$ is called standard character.

Theorem 2.11. [12] Let $G$ be 2-transitive group, then the standard character of $G$ is irreducible character.

Theorem 2.12. [6] Let $G$ be a finite group with a normal symmetric subset $S$. Let $A$ be the adjacency matrix of graph $\operatorname{Cay}(G, S)$. Then the eigenvalues of $A$ are given by

$$
\left[\lambda_{\chi}\right]^{\chi(1)^{2}}, \chi \in \operatorname{Irr}(G)
$$

where $\lambda_{\chi}=\frac{1}{\chi(1)} \sum_{s \in S} \chi(s)$.

Theorem 2.13. The derangement graph of any 2-transitive group is not a bipartite graph.

Proof. Let $G$ acts 2-transitive on $n$ elementsa and complete bipartite graph $K_{r, s}$ be the derangement graph of $G$. Since the derangement graph is a regular graph, we have $r=s$. The eigenvalues of $K_{r, r}$ are $\left\{[-r]^{1},[0]^{2 r-2},[r]^{1}\right\}$. On the other hand by Theorem 2.11, the standard character $\pi$ of a 2-transitive group is irreducible. So by Theorem 2.12, we have $\lambda_{\chi}=\frac{-|\mathcal{D}|}{\chi(1)}=\frac{-r}{n-1}$. Since the rational eigenvalues of a graph are integers, we have $n=2$ and then $G \cong \mathbb{Z}_{2}$ or $G \cong\{1\}$.

\section{Partial difference set}

Let $G$ be a finite group and $D \subseteq G$. Then $D$ is a $(n, k, \lambda, \mu)$-partial difference set (PDS) in $G$ if and only if $D D^{-1}=\gamma 1_{G}+\lambda D+\mu(G-D)$, where $\gamma=k-\mu$ if $1_{G} \notin D$ and $\gamma=k-\lambda$ if $1_{G} \in D$. We will usually assume that $1_{G} \notin D$ and $D^{(-1)}=D$, in which case, we have

$$
D^{2}=(k-\mu) 1_{G}+(\lambda-\mu) D+\mu G .
$$

Partial difference sets were named by I. M. Chakravarti, 1969 [4], but introduced by Bose and Cameron, 1965 [3] in their studies of calibration designs and the bridge tournament problem. $D$ is called abelian if $G$ is abelian. It is well known that a PDS $D$ with $1 \notin D$ and $\left\{d^{-1}: d \in D\right\}=D$ is equivalent to a strongly regular Cayley graph, such a PDS is called regular. The study of partial difference sets is closely related to partial geometries, Schur rings, strongly regular Cayley graphs and two-weight codes. Asurvey of Ma [15] contains very detailed descriptions of these connections. 
Theorem 3.1. Let $G=H K \leq \operatorname{Sym}(n)$ be a Frobenius group with kernel $K$. The derangement set of $G$ is a $(n|H|, n-1, n-2,0)-P D S$.

Proof. We know that $|K|=n$. Every non-identity element of kernel $G$ is a derangement of $G$ and $\mathcal{D} \cup\{1\}$ is a subgroup. This implies that the derangement set of $G$ is a $(n|H|, n-1, n-2,0)-\mathrm{PDS}$.

Theorem 3.2. Consider the dihedral group $D_{2 n}$ with derangement set $\mathcal{D}$. If $n$ is odd, then $\mathcal{D}$ is a PDS and if $n$ is even, then $\mathcal{D}$ is not a PDS.

Proof. Consider the dihedral group $D_{2 n}=\left\langle a, b \mid a^{n}=b^{2}=1, a b a^{-1}=a^{-1}\right\rangle$. If $n$ is odd, then $D_{2 n}$ is a Frobenius group and by Theorem 3.1 the derangement set is a PDS. Now, let $n$ be even. Suppose that $a=(1,2,3, \ldots, n)$ and $b=(1,2)(3, n) \ldots\left(\frac{n}{2}+1, \frac{n}{2}+2\right)$ is permutation presentation of generators of $D_{2 n}$. The derangement set of $D_{2 n}$ is

$$
\mathcal{D}=\left\{a, a^{2}, \ldots, a^{n-1}, b, a^{2} b, a^{4} b, \ldots, a^{n-2} b\right\} .
$$

If $a^{i} a^{-j}=a^{2}$, then $i-j \equiv 2(\bmod n)$ and $\{(3,1),(4,2), \ldots,(n-1, n-3)\}$ are $n-3$ solutions for $(i, j)$. On the other hand, if $\left(a^{i} b\right)\left(a^{j} b\right)^{-1}=a^{2}(i, j$ are even), then $a^{i} a^{-j}=a^{2}$ and so $i-j \equiv 2(\bmod n)$. Thus $\{(4,2),(6,4), \ldots,(n-2, n-4)\}$ are $n / 2-2$ solutions for $(i, j)$. One can see that $a\left(a^{n-1}\right)^{-1}=a^{2}, b\left(a^{n-2} b\right)^{-1}=a^{2}$ and $\left(a^{2} b\right) b^{-1}=a^{2}$. Let $\left(a^{i} b\right) a^{-j}=a^{2}$, by using the relation of group, we have $a^{i-j} b=a^{2}$ and this is impossible. The equation $a^{i}\left(a^{j} b\right)^{-1}=a^{2}$ is impossible, too. So if $d_{i}, d_{j} \in \mathcal{D}$, then $d_{i} d_{j}^{-1}=a^{2}$ has $(3 n / 2)-2$ solutions. If $a^{i} a^{-j}=a$, then $i-j \equiv 1(\bmod n)$ and $\{(2,1),(3,2), \ldots,(n-1, n-2)\}$ are the solutions for $(i, j)$. By the relation of $D_{2 n}$, there is no other solutions for $d_{i} d_{j}^{-1}=a$. So in this case there are $n-2$ solutions. Then we conclude that the derangement set of dihedral group in this case is not a PDS.

Consider the dicyclic group $T_{4 n}, U_{6 n}$ and $V_{8 n}$ by the following presentations:

$$
\begin{aligned}
& T_{4 n}=\left\langle a, b \mid a^{2 n}=e, a^{n}=b^{2}, b^{-1} a b=a^{-1}\right\rangle, \\
& U_{6 n}=\left\langle a, b \mid a^{2 n}=b^{3}=e, a^{n}=b^{2}, a^{-1} b a=b^{-1}\right\rangle, \\
& V_{8 n}=\left\langle a, b \mid a^{2 n}=b^{4}=e, a b a=b^{-1}, a b^{-1} a=b^{-1}\right\rangle .
\end{aligned}
$$

Theorem 3.3. The derangement set of dicyclic group $T_{4 n}$ is a $(4 n, 4 n-1,4 n-$ $2,0)-P D S$.

Proof. In [7] Darafsheh proved that two elements $a=(1,2,3, \ldots, 2 n)(2 n+1,2 n+$ $2,2 n+3, \ldots, 4 n)$ and $b=(1,2 n+1, n+1,3 n+1)(2,4 n, n+2,3 n)(3,4 n-1 n+$ $3,3 n-1), \ldots,(n-1,3 n+3,2 n-1,2 n+3)(n, 3 n+2,2 n, 2 n+2)$ are the generators of $T_{4 n}$. All elements of $T_{4 n}$ have no fixed point. Then $\mathcal{D}=T_{4 n}-\{e\}$ which is a $(4 n, 4 n-1,4 n-2,0)-\mathrm{PDS}$. 
Theorem 3.4. The derangement set of $U_{6 n}(n \geq 4)$ is not a PDS set.

Proof. Let $a=(1,2,3, \ldots, 2 n)(2 n+1,2 n+2)$ and $b=(2 n+1,2 n+2,2 n+3)$ be the permutation peresentations of generators of $U_{6 n}$ [7]. One can see that the derangement set of $U_{6 n}$ is $\mathcal{D}=\left\{a^{i} b, a^{i} b^{2} \mid 2 \leq i \leq 2 n-2\right.$ and $i$ is even $\}$. Let $a^{i} b^{j}, a^{r} b^{s} \in \mathcal{D}$ and $\left(a^{i} b^{j}\right)\left(a^{r} b^{s}\right)^{-1}=b$. Then we have $a^{i} b^{j-s} a^{-r}=b$ and so $a^{-i} b a^{r}=b^{j-s}$. Thus $a^{r-i} a^{-r} b a^{r}=b^{j-s}$ and by using the relation of $U_{6 n}$, we have $a^{r-i} b^{(-1)^{r}}=b^{j-s}$. This yields that

$$
\left\{\begin{array}{l}
r \equiv i(\bmod 2 n) \\
j-s=1
\end{array} .\right.
$$

Hence the relation $\left(a^{i} b^{j}\right)\left(a^{r} b^{s}\right)^{-1}=b$ has $n-1$ solutions. On the other hand $\left(a^{i} b^{j}\right)\left(a^{r} b^{s}\right)^{-1}=a$ has no solution and thus $\mathcal{D}$ is not a PDS set.

Theorem 3.5. The derangement set of $V_{8 n}(n \geq 3)$ is not a PDS set.

Proof. For group $V_{8 n}$ we can consider two following cases:

- Case 1. Suppose $n$ is an odd number. Let $a=(1,2,3, \ldots, 2 n)(2 n+1,2 n+$ $2, \ldots, 4 n)$ and $b=(1,2,2 n+1,2 n+2)(3,2 n, 2 n+3,4 n)(4,4 n-1,2 n+4,2 n-$ 1) $\ldots(n+1,3 n+2,3 n+1, n+2)$ be the permutation peresentations of generators of $V_{8 n}$ [7]. One can see that the derangement set of $V_{8 n}$ is

$$
\mathcal{D}=\left\{a, a^{2}, \ldots, a^{2 n-1}, b, b^{2}, b^{3}, a^{i} b, a^{i} b^{2}, a^{i} b^{3}, a^{r} b^{2}\right\},
$$

where $2 \leq i \leq 2 n-2$ ( $i$ is even) and $1 \leq r \leq 2 n-1$ ( $r$ is odd $)$.

We are going to show that the number of elements of $A=\left\{d_{i}, d_{j} \in \mathcal{D} \mid d_{i} d_{j}^{-1}=a\right\}$ and $B=\left\{d_{i}, d_{j} \in \mathcal{D} \mid d_{i}, d_{j}^{-1}=a^{2}\right\}$ are not equal. By considering $i-j \equiv$ $1(\bmod 2 n)$, the equation $a^{i}\left(a^{j}\right)^{-1}=a$ has $2 n-2$ solutions. Similarly, the equation $\left(a^{i} b^{2}\right)\left(a^{j} b^{2}\right)^{-1}=a$ has $2 n-2$ solutions. On the other hand, we have $b^{2}\left(a^{2 n-1} b^{2}\right)^{-1}=a$ and $\left(a b^{2}\right)\left(b^{2}\right)^{-1}=a$. So the set $A$ has $4 n-2$ elements. Now, we compute the elements of the set $B$. By considering $i-j \equiv 2(\bmod 2 n)$, the equation $a^{i}\left(a^{j}\right)^{-1}=a^{2}$ has $2 n-3$ solutions. Also, $\left(a^{i} b^{2}\right)\left(a^{j} b^{2}\right)^{-1}=a^{2}$ has $2 n-3$ solutions. Suppose that $4 \leq i \leq 2 n-2(i$ is even $)$ and $j \equiv i-2(\bmod 2 n)$, then we have $\left(a^{i} b\right)\left(a^{j} b\right)^{-1}=a^{2}$ and $\left(a^{i} b^{3}\right)\left(a^{j} b^{3}\right)^{-1}=a^{2}$. This means that each of this equations has $n-2$ solutions. On can see that $b^{i}\left(a^{2 n-2} b^{i}\right)^{-1}=a^{2}$ for $i=1,2,3$. On the other hand, we have $\left(a^{2} b^{i}\right)\left(b^{-i}\right)=a^{2}(i=1,2,3),\left(a b^{2}\right)\left(a^{2 n-1} b^{2}\right)=a^{2}$ and $a\left(a^{2 n-1}\right)^{-1}=a^{2}$. Then the set $B$ has $6 n-2$ elements and the derangement set of $V_{8 n}(n$ is odd $)$ is not a PDS set.

- Case 2. Suppose $n$ is even number. Let $a=(1,2,3, \ldots, 2 n)(2 n+1,2 n+$ $2, \ldots, 4 n)$ and $b=(1,2,2 n+1,2 n+2)(3,2 n, 2 n+3,4 n)(4,4 n-1,2 n+4,2 n-$ 1) $\ldots(n, 3 n+3,3 n, n+3)(n+1, n+2,3 n+1,3 n+2)$ be the permutation peresentations of generators of $V_{8 n}$ [7]. One can see that the derangement set of $V_{8 n}$ is

$$
\mathcal{D}=\left\{a, a^{2}, \ldots, a^{2 n-1}, b, b^{2}, b^{3}, a^{i} b, a^{i} b^{2}, a^{i} b^{3}, a^{r} b, a^{r} b^{2}, a^{s} b^{2}, a^{s} b^{3}\right\},
$$


where $2 \leq i \leq 2 n-2(i$ is even $), r \in\{1,5,9, \ldots, 2 n-3\}$ and $s \in\{3,7,11, \ldots, 2 n-1\}$.

Now, we show that the number of elements of $E=\left\{d_{i}, d_{j} \in \mathcal{D} \mid d_{i} d_{j}^{-1}=a\right\}$ and $F=\left\{d_{i}, d_{j} \in \mathcal{D} \mid d_{i} d_{j}^{-1}=a^{4}\right\}$ are not equal. By regarding $i-j \equiv 1(\bmod 2 n)$, the equation $a^{i}\left(a^{j}\right)^{-1}=a$ has $2 n-2$ solutions. If $j \equiv i-1(\bmod n)$ and $i \in$ $\{2,5,6,9,10, \ldots, 2 n-2\}$, then the equation $\left(a^{i} b^{s}\right)\left(a^{j} b^{s}\right)^{-1}=a$, where $s \in\{1,2\}$ has $n-1$ solutions. If $j \equiv i-1(\bmod n)$ and $i \in\{3,4,7,8,11, \ldots, 2 n-1\}$, then the equation $\left(a^{i} b^{s}\right)\left(a^{j} b^{s}\right)^{-1}=a$, where $s \in\{2,3\}$ has $n-1$ solutions. One can see that $\left(a b^{t}\right)\left(b^{t}\right)^{-1}=a$, where $t \in\{1,2\}$ and $b^{t}\left(a^{2 n-1} b^{t}\right)^{-1}=a$, where $t \in\{2,3\}$. Then the set $E$ has $6 n-2$ elements. Now, we compute the elements of the set $F$. By considering $i-j \equiv 4(\bmod 2 n)$ the equation $a^{i}\left(a^{j}\right)^{-1}=a^{4}$ has $2 n-5$ solutions. It is clear that $a^{1}\left(a^{2 n-3}\right)^{-1}=a^{2}\left(a^{2 n-2}\right)^{-1}=a^{3}\left(a^{2 n-1}\right)^{-1}=a^{4}$. One can see that if $t \in\{1,2,3\}$ then $\left(a^{4} b^{t}\right)\left(b^{t}\right)^{-1}=a^{4}$, and $b^{t}\left(a^{2 n-4} b^{t}\right)^{-1}=a^{4}$. Let $i, j$ be even, $i-j \equiv 4(\bmod 2 n)$ and $r \in\{1,2,3\}$. Then $\left(a^{i} b^{r}\right)\left(a^{j} b^{r}\right)^{-1}=a^{4}$ yields $3(n-1)$ solutions. Let $i$ be odd, $i-j \equiv 4(\bmod 2 n)$ and $r \in\{5,9,13, \ldots, 2 n-3\}$. Then by using $\left(a^{i} b^{r}\right)\left(a^{j} b^{r}\right)^{-1}=a^{4}$ we get $n-2$ solutions for this equation. Let $i$ be odd, $i-j \equiv 4(\bmod 2 n)$ and $r \in\{7,11,15, \ldots, 2 n-1\}$. Again by $\left(a^{i} b^{r}\right)\left(a^{j} b^{r}\right)^{-1}=a^{4}$ we acheive $n-2$ solutions. If $i \in\{1,2\}$ then $\left(a b^{i}\right)\left(a^{2 n-3} b^{i}\right)^{-1}=a^{4}$. If $i \in\{2,3\}$ then $\left(a^{3} b^{i}\right)\left(a^{2 n-1} b^{i}\right)^{-1}=a^{4}$ and if $i \in\{1,2,3\}$ then $\left(a^{2} b^{i}\right)\left(a^{2 n-2} b^{i}\right)^{-1}=a^{4}$. So the set $F$ has $7 n-2$ elements. Then the derangement set of $V_{8 n}(n$ is odd) is not a PDS set.

\section{REF EREN CES}

1. B. Ahmadi: Maximum intersecting families of permutations. Ph.D. thesis. University of Regina, Regina, 2013.

2. B. Ahmadi and K. Meagher: The Erdös-Ko-Rado property for some permutation groups. Austr. J. Comb. 61 (2015), 23-41.

3. R. C. Bose and J. M. Cameron: The bridge tournament problem and calibration designs for comparing pairs of objects. Journal of Research of the NBS-B, Maths. and Math.Phys. 69 (1965), 323-332.

4. I. M. Chakravarti: Partial difference sets, calibration designs and error correcting codes. Bull. Inter. Stat. Inst. 43 (1969), 104-106.

5. J. D. Dixon and B. Mortimer: Permutation Groups. Springer, 1966.

6. P. Diaconis and M. Shahshahani: Generating a random permutation with random transpositions. Zeit. für Wahrscheinlichkeitstheorie verw. Gebiete. 57 (1981), $159-179$.

7. M. R. Darafsheh and N. S. Poursalavati: On the existence of the orthogonal basis of the symmetry classes of tensors associated with certain groups. SUT J. Math. 37 (2001), 1-17. Zbl 0999.20006

8. P. Erdös, Chao Ko and R. Rado: Intersection theorems for systems of finite sets. Quart. J. Math. 12 (1961), 313--320.

9. P. Frankl and M. Deza: The maximum number of permutations with given maximal or minimal distance. J. Combin. Theory Ser. A. 22 (1977), 352--360. 
10. M. Ghorbani and M. Rajabi-Parsa: On the Erdös-Ko-Rado property of finite groups of order a product of primes. Electronic journal of graph theory and applications. 7 (2019), 31-42.

11. M. Ghorbani and M. Rajabi-Parsa: On the spectrum of derangement graphs of order a product of three primes. 6 (2019), 81-89.

12. I. M. Isaacs: Character Theory of Finite Groups. University of Wiaconslr. New York. Academic Press, 1976.

13. M. Jalali-Rad and A. R. Ashrafi: Erdös-Ko-Rado properties of some finite groups. Sib. Electron. Math. Reports. 13 (2016), 1249-1257.

14. G. James and M. Liebeck: Representation and characters of groups. Cambridge University Press. Cambridge, 1993.

15. S. L. Ma: A survey of partial difference sets. Designs, Codes and Cryptogr. 4 (1994), 221-261.

16. J.S. Rose: A Course on Group Theory. Cambridge University Press, 1978.

17. J. Wang and S. J. Zhang: An Erdös-Ko-Rado-type theorem in Coxeter groups. European J. Combin. 29 (2008), 1112-1115.

Modjtaba Ghorbani

Department of Mathematics

Faculty of Science

Shahid Rajaee Teacher Training University

Tehran, 16785-136, I. R. Iran

mghorbani@sru.ac.ir

Mina Rajabi-Parsa

Department of Mathematics

Faculty of Science

Shahid Rajaee Teacher Training University

Tehran, 16785-136, I. R. Iran

mina.rparsa@gmail.com 